\title{
Efficient Blind Receiver Design for Orthogonal Space-Time Block Codes
}

\author{
Tao Cui, Student Member, IEEE, and Chintha Tellambura, Senior Member, IEEE
}

\begin{abstract}
We consider stochastic blind maximum-likelihood detection of orthogonal space-time block codes (OSTBCs) over a quasi-static flat multiple-input multiple-output (MIMO) Rayleigh fading channel. A general decision rule for stochastic blind maximum-likelihood OSTBC detection is derived. This rule is simplified using OSTBC linear dispersion matrices to realize a blind detector, which is implemented by semi-definite relaxation or sphere decoding. For the latter, the modifications necessary for both unitary and non-unitary constellations are developed. Two totally blind detectors using dual constellations or a superimposed training scheme are proposed. As a side product, two conditions for a rotatable OSTBC are also derived. A decision-directed, minimum mean-square-error (MMSE) channel estimator is developed. We also derive the Cramér-Rao bound (CRB) for channel estimation and discuss the optimal power allocation. Extensive simulation results are used to compare the different detectors in terms of complexity and performance.
\end{abstract}

Index Terms-Blind detector, channel estimation, maximumlikelihood, MIMO, OSTBC.

\section{INTRODUCTION}

$\mathbf{O}$ RTHOGONAL space-time block codes (OSTBCs) are a popular multiple antenna technology [1]-[3]. They achieve full transmit diversity and are amenable to simple linear maximum-likelihood (ML) detection if the channel state information (CSI) is known at the receiver. However, CSI estimation for a multiple antenna channel requires the transmission of pilot symbols, reducing the effective data rate. Moreover, the linear ML detector is contingent upon a static channel over the length of the entire block. ${ }^{1}$ The channel variation destroys the orthogonality of the OSTBC receiver filter, and the linear ML detector will no longer be optimal [4]-[6]. Even when the channel is static over each signal block but varies from block to block, it is difficult to track the time varying channel. These factors have motivated the

Manuscript received September 8, 2005; revised April 20, 2006, November 2, 2006, and November 21, 2006; accepted November 22, 2006. The associate editor coordinating the review of this paper and approving it for publication was G. Leus. This work has been supported in part by the Natural Sciences and Engineering Research Council of Canada, Informatics Circle of Research Excellence and Alberta Ingenuity Fund. This paper has been presented in part at IEEE Wireless Communications and Networking Conference, April 2006, Las Vegas, NV USA.

T. Cui was with the Department of Electrical and Computer Engineering, University of Alberta, Edmonton, AB T6G 2V4, Canada. He is now with the Department of Electrical Engineering, California Institute of Technology, Pasadena, CA 91125 USA (e-mail: taocui@ caltech.edu).

C. Tellambura is with the Department of Electrical and Computer Engineering, University of Alberta, Edmonton, AB, Canada T6G 2V4 (e-mail: chintha@ece.ualberta.ca).

Digital Object Identifier 10.1109/TWC.2007.05709.

${ }^{1}$ Throughout this paper, the term 'block' refers to an OSTBC signal matrix (i.e., codeword). development of blind and semi-blind OSTBC detectors [7][11].

In [7], an approximate blind ML OSTBC detector is developed via a suboptimal blind detector (cyclic detector). Reference [8] develops subspace based blind and semi-blind detectors. References [9]-[11] develop a deterministic blind ML detector for binary phase shift keying (BPSK) or quadrature phase shift keying (QPSK) OSTBCs, using semi-definite relaxation (SDR) and sphere decoding. The resulting detectors perform substantially better in terms of the bit error rate (BER) than the previous blind detectors [7], [8]. These studies assume a static channel (i.e., one that is constant over several signalling blocks) and hence neglect any consideration of the Doppler rate - implicity assuming a zero Doppler rate.

In this paper, different from [7]-[11], we consider stochastic blind ML OSTBC detection for a quasistatic fading channel, i.e., the channel remains constant for a single block only and varies from block to block. ${ }^{2}$ This model is valid for normalized Doppler rates up to $3 \%$ [12]. We derive a general decision rule for stochastic ML blind OSTBC detection and show that it leads to a discrete quadratic optimization problem. Although such problems can be solved via exhaustive search when the size is small, we develop the detectors based on sphere decoding [13], [14], which provides the same optimal solution as exhaustive search but with much less computational complexity, and semi-definite relaxation [15]. For sphere decoding, the modifications necessary for both unitary and non-unitary constellations are developed. We note that [9]-[11] also develop detectors based on semi-definite relaxation and sphere decoding. The differences are that our detectors are developed for arbitrary constellations, hold for quasistatic channels, and take a stochastic approach. For the latter reason, the term stochastic will be used throughout this paper. Although the inherent phase ambiguity of blind detectors can be overcome by a few pilot symbols [7], [9]-[11], which however lowers the bandwidth efficiency, we present two novel approaches for totally blind detection without the use of explicit pilot symbols. The first scheme uses dual constellations such that the phase angle of any element of the first constellation is different from that of any element in the second constellation. As a side product, two conditions for a rotatable OSTBC are also derived. The second scheme utilizes superimposed training, where pilot symbols are added to data symbols. We derive a decision-directed, minimum mean-square-error (MMSE) channel estimator to alleviate the computational burden of the blind detectors. We also derive the Cramér-Rao

\footnotetext{
${ }^{2}$ However, for a more realistic assessment, in our simulations, we assume a continuous fading channel with the Jakes' spectrum and a given Doppler.
} 
bound (CRB) for channel estimation and discuss the optimal power allocation. The blind detector and the channel estimator form an efficient OSTBC receiver structure (Fig. 1). Extensive simulation results are used to compare the different detectors in terms of complexity and performance.

The rest of the paper is organized as follows. The system model and OSTBCs are introduced in Section II. Section III derives the general stochastic blind ML detection rule and develops SRD and the sphere decoder based blind detectors. Section IV presents the two totally blind schemes. Section V gives the MMSE channel estimator and the CRB. Simulation results are given in Section VI and conclusions are drawn in Section VII.

Notation: $E\{\cdot\},(\cdot)^{*},(\cdot)^{T},(\cdot)^{H}$ and $(\cdot)^{\dagger}$ denote expectation, complex conjugation, transpose, conjugate transpose and Moore-Penrose pseudo-inverse, respectively. The imaginary unit is $\jmath=\sqrt{-1}$. The trace, determinant and the squared Frobenius norm of matrix $\mathbf{A}$ are $\operatorname{tr}(\mathbf{A}), \operatorname{det}(\mathbf{A})$ and $\|\mathbf{A}\|_{F}=$ $\sqrt{\operatorname{tr}\left(\mathbf{A A}^{H}\right)}$. vec $(\mathbf{A})$ denotes the vector formed by stacking the columns of A. A circularly complex Gaussian variable with mean $\mu$ and variance $\sigma^{2}$ is denoted by $z \sim \mathcal{C N}\left(\mu, \sigma^{2}\right)$. The sets of real numbers and integers are $\mathbb{R}$ and $\mathbb{Z}$. The $N \times N$ identity matrix is $\mathbf{I}_{N}$. The Kronecker delta is $\delta_{i, j}=1$ if $i=j$ and $\delta_{i, j}=0$ if $i \neq j$ where $i, j \in \mathbb{Z} . \mathbf{A} \succeq \mathbf{B}$ denotes that $\mathbf{A}-\mathbf{B}$ is positive semi-definite.

\section{SySTEM MODEL}

We consider a MIMO system with $N_{t}$ transmit and $N_{r}$ receive antennas. Each block of transmitted symbols occupies $T$ time slots and time interval $T_{B}$. The symbols transmitted during the $n$th block are denoted by the $T \times N_{t}$ matrix $\mathbf{S}[n]=$ $\left[s_{t, i}[n]\right], t=1,2, \ldots, T$ and $i=1,2, \ldots, N_{t}$, where $s_{t, i}[n]$ is transmitted by the $i$ th antenna in the $t+(n-1) T$-th time slot. For an OSTBC, $P$ symbols $\mathbf{x}[n]=\left[x_{1}[n], x_{2}[n], \ldots, x_{P}[n]\right]^{T}$ with the same average power $E_{s}=E\left\{\left|x_{p}[n]\right|^{2}\right\}$ form $\mathbf{S}[n]$. The entries of $\mathbf{S}[n]$ are linear in $x_{i}[n]$ and $x_{i}^{*}[n]$, and the block has the orthogonal property

$$
\mathbf{S}^{H}[n] \mathbf{S}[n]=c\left(\sum_{p=1}^{P}\left|x_{p}[n]\right|^{2}\right) \mathbf{I}_{N_{t}},
$$

where $c=1 / r$ and $r=P / T$ is the rate of the code. For the Alamouti code [1] or the $\mathcal{G}_{2}$ code in [2], $N_{t}=2, P=2$, $T=2, c=1$ and $\mathbf{S}[n]$ is given by

$$
\mathbf{S}[n]=\left(\begin{array}{cc}
x_{1}[n] & x_{2}[n] \\
-x_{2}^{*}[n] & x_{1}^{*}[n]
\end{array}\right) .
$$

For the $\mathcal{G}_{3}$ and $\mathcal{G}_{4}$ codes in [2], $c=2$.

An OSTBC can be alternately represented as [16]

$$
\begin{aligned}
\mathbf{S}[n] & =\sum_{p=1}^{P}\left(\alpha_{p}[n] \mathbf{A}_{p}+\jmath \beta_{p}[n] \mathbf{B}_{p}\right) \\
& =\sum_{p=1}^{P}\left(x_{p}[n] \mathbf{C}_{p}+x_{p}^{*}[n] \mathbf{D}_{p}\right),
\end{aligned}
$$

where $x_{p}[n]=\alpha_{p}[n]+\jmath \beta_{p}[n]$ and $\mathbf{A}_{q}, \mathbf{B}_{q}$ are called dispersion matrices, a set of constant matrices that defines an OSTBC [16]. This representation (3) will be used later.

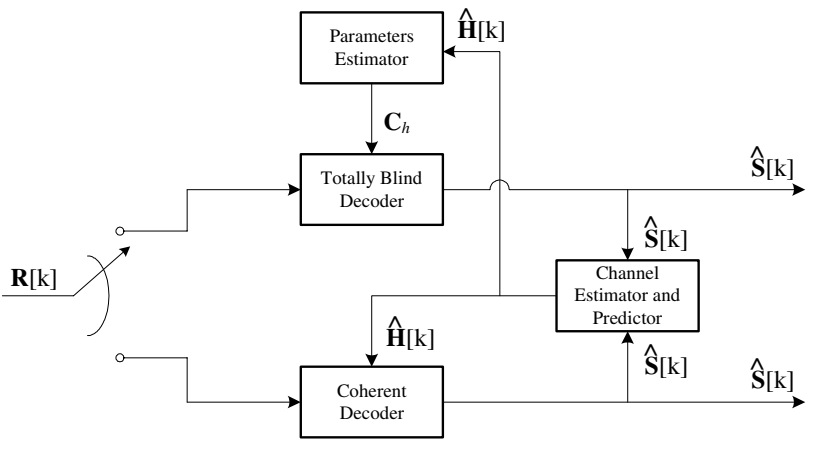

Fig. 1. The block diagram of blind receiver for OSTBC.

We consider a frequency-flat Rayleigh fading MIMO channel resulting from a rich scattering environment. The received signal at the $j$ th receive antenna at time slot $t$ in the $n$th block is

$$
r_{t, j}[n]=\sum_{i=1}^{N_{t}} h_{i, j}[n] s_{t, i}[n]+w_{t, j}[n],
$$

where $h_{i, j}[n]$ denotes the path gain from the $i$ th transmit antenna to the $j$ th receive antenna, and $w_{t, j}[n]$ is the complex additive white Gaussian noise at the $j$ th receive antenna with mean zero and variance $\sigma_{n}^{2}$. The fading channel is assumed to be quasistatic, i.e., channel variations within each block are negligible. All path gains are statistically independent $\left(E\left\{h_{i, j}[n] h_{i^{\prime}, j^{\prime}}^{*}[n]\right\}=\delta_{i, i^{\prime}} \delta_{j, j^{\prime}}\right)$ and have the same time correlation function $R_{h}(\tau)$. Typically, when classical Jakes' model [17] is used, $R_{h}[m]$ is given by

$R_{h}[m]=E\left\{h_{i, j}[n] h_{i^{\prime}, j^{\prime}}^{*}[n+m]\right\}=\delta_{i, i^{\prime}} \delta_{j, j^{\prime}} \sigma_{h}^{2} J_{0}\left(2 \pi m f_{d} T_{B}\right)$,

where $\sigma_{h}^{2}$ denotes the power of the path gain, $J_{0}(\cdot)$ is the zeroth order Bessel function of the first kind, and $f_{d}$ is the Doppler frequency. Note that the quasistatic condition holds when $f_{d} T_{B}<0.03$. The matrix version of (4) is

$$
\mathbf{R}[n]=\mathbf{S}[n] \mathbf{H}[n]+\mathbf{W}[n],
$$

where $\mathbf{R}[n]=\left[r_{t, j}[n]\right]$ is the $T \times N_{r}$ receive matrix, $\mathbf{H}[n]=$ $\left[h_{i, j}[n]\right]$ is the $N_{t} \times N_{r}$ channel matrix, and $\mathbf{W}[n]=\left[w_{t, j}[n]\right]$ is the $T \times N_{r}$ noise matrix.

\section{MAXimum-Likelihood Blind Decoding}

This section derives the general ML metric for stochastically blind OSTBC detection using $N$ consecutive received signal blocks. This rule will be used for the totally blind detectors in Section IV or the totally blind detector block in Fig. 1. We consider the received blocks during $n=k+1$ to $n=k+N$. Let $\overline{\mathbf{R}}[k]=\left[\mathbf{R}^{T}[k+1], \mathbf{R}^{T}[k+2], \ldots, \mathbf{R}^{T}[k+N]\right]^{T}$ and $\overline{\mathbf{S}}[k]=\left[\mathbf{S}^{T}[k+1], \mathbf{S}^{T}[k+2], \ldots, \mathbf{S}^{T}[k+N]\right]^{T}$. The stochastic ML decision rule for the sequence $\overline{\mathbf{S}}[k]$ can be expressed as

$$
\hat{\overline{\mathbf{S}}}[k]=\underset{\mathbf{S}[k]}{\arg \max } f(\overline{\mathbf{R}}[k] \mid \overline{\mathbf{S}}[k]),
$$

where $f(a \mid b)$ is the probability density function (pdf) of $a$ conditioned on $b$. The conditional pdf (7) can be calculated by 
averaging the pdf $f(\overline{\mathbf{R}}[k] \mid \overline{\mathbf{S}}[k], \overline{\mathbf{H}}[k])$ over the channel matrix $\overline{\mathbf{H}}[k]$, which results in

$$
f(\overline{\mathbf{R}}[k] \mid \overline{\mathbf{S}}[k])=\frac{\exp \left(-\operatorname{tr}\left(\overline{\mathbf{R}}^{H}[k] \mathbf{C}_{R}^{-1}[k] \overline{\mathbf{R}}[k]\right)\right)}{\left(\pi^{N N_{t}} \operatorname{det}\left(\mathbf{C}_{R}[k]\right)\right)^{N_{r}}},
$$

where the conditional covariance matrix $\mathbf{C}_{R}[k]$ is given by

$$
\begin{aligned}
\mathbf{C}_{R}[k] & =E\left\{\overline{\mathbf{R}}[k] \overline{\mathbf{R}}^{H}[k] \mid \overline{\mathbf{S}}[k]\right\} \\
& =\overline{\mathbf{S}}_{D}[k] \mathbf{C}_{H} \overline{\mathbf{S}}_{D}^{H}[k]+N_{r} \sigma_{n}^{2} \mathbf{I}_{T N},
\end{aligned}
$$

where $\overline{\mathbf{S}}_{D}[k]$ is a block diagonal matrix

$$
\overline{\mathbf{S}}_{D}[k]=\left[\begin{array}{llll}
\mathbf{S}[k+1] & & & \\
& \mathbf{S}[k+2] & & \\
& & \ddots & \\
& & & \mathbf{S}[k+N]
\end{array}\right]
$$

and $\mathbf{C}_{H}$ is the covariance matrix of the vector $\overline{\mathbf{H}}=\left[\mathbf{H}^{T}[k+\right.$ $\left.1], \mathbf{H}^{T}[k+2], \ldots, \mathbf{H}^{T}[k+N]\right]^{T} . \mathbf{C}_{H}$ can be represented as

$$
\mathbf{C}_{H}=N_{r}\left(\mathbf{C}_{h} \otimes \mathbf{I}_{N_{t}}\right)
$$

where $\otimes$ denotes the Kronecker product and $\mathbf{C}_{h}$ is given by

$$
\mathbf{C}_{h}=\left[\begin{array}{cccc}
R_{h}[0] & R_{h}[1] & \cdots & R_{h}[N-1] \\
R_{h}[-1] & R_{h}[0] & \vdots & \vdots \\
\vdots & \vdots & \ddots & \vdots \\
R_{h}[-N+1] & \cdots & \cdots & R_{h}[0]
\end{array}\right]
$$

If $x_{p}[n]$ 's belong to a unitary constellation, we have

$$
\mathbf{S}^{H}[n] \mathbf{S}[n]=T E_{s} \mathbf{I}_{N_{t}} .
$$

However, if $x_{p}[n]$ 's are from a non-unitary constellation, when $P$ is large (for example, $P \geq 4$ ), $\sum_{p=1}^{P}\left|x_{p}[n]\right|^{2} \approx P E_{s}$ applying the law of large numbers to (1) and we have

$$
\mathbf{S}^{H}[n] \mathbf{S}[n] \approx T E_{s} \mathbf{I}_{N_{t}} .
$$

Since $\operatorname{det}\left(\mathbf{C}_{R}[k]\right)=\operatorname{det}\left(\mathbf{C}_{H} \overline{\mathbf{S}}_{D}^{H}[k] \overline{\mathbf{S}}_{D}[k]+N_{r} \sigma_{n}^{2} \mathbf{I}_{N_{t} N}\right) \approx$ $\operatorname{det}\left(T E_{s} \mathbf{C}_{H} \mathbf{I}_{N_{t} N}+N_{r} \sigma_{n}^{2} \mathbf{I}_{N_{t} N}\right)$ is almost independent of $\overline{\mathbf{S}}_{D}[k]$ for both unitary and non-unitary constellations, (7) is equivalent to

$$
\overline{\mathbf{S}}[k]=\underset{\mathbf{S}[k]}{\arg \min } \operatorname{tr}\left(\overline{\mathbf{R}}^{H}[k] \mathbf{C}_{R}^{-1}[k] \overline{\mathbf{R}}[k]\right) .
$$

Using the identity $(\mathbf{A}+\mathbf{B C D})^{-1}=\mathbf{A}^{-1}-\mathbf{A}^{-1} \mathbf{B}\left(\mathbf{C}^{-1}+\right.$ $\left.\mathbf{D A}^{-1} \mathbf{B}\right)^{-1} \mathbf{D A}^{-1}$, (15) becomes

$$
\begin{aligned}
\hat{\overline{\mathbf{S}}}[k]= & \underset{\mathbf{\mathbf { S }}[k]}{\arg \min } \frac{1}{N_{r} \sigma_{n}^{2}} \operatorname{tr}\left(\overline { \mathbf { R } } ^ { H } [ k ] \left(\mathbf{I}_{T N}-\overline{\mathbf{S}}_{D}[k]\right.\right. \\
& \left.\left.\times\left(N_{r} \sigma_{n}^{2} \mathbf{C}_{H}^{-1}+\overline{\mathbf{S}}_{D}^{H}[k] \overline{\mathbf{S}}_{D}[k]\right)^{-1} \overline{\mathbf{S}}_{D}^{H}[k]\right) \overline{\mathbf{R}}[k]\right) \\
= & \underset{\mathbf{\mathbf { S }}[k]}{\arg \max } \operatorname{tr}\left(\begin{array}{c}
\overline{\mathbf{R}}^{H}[k] \overline{\mathbf{S}}_{D}[k]\left(N_{r} \sigma_{n}^{2} \mathbf{C}_{H}^{-1}\right. \\
\left.+\overline{\mathbf{S}}_{D}^{H}[k] \overline{\mathbf{S}}_{D}[k]\right)^{-1} \overline{\mathbf{S}}_{D}^{H}[k] \mathbf{\mathbf { R }}[k]
\end{array}\right) \\
= & \underset{\mathbf{\mathbf { S }}[k]}{\arg \max } \operatorname{tr}\left(\overline{\mathbf{R}}^{H}[k] \overline{\mathbf{S}}_{D}[k] \mathbf{C} \overline{\mathbf{S}}_{D}^{H}[k] \overline{\mathbf{R}}[k]\right),
\end{aligned}
$$

where $\mathbf{C}=\left(N_{r} \sigma_{n}^{2} \mathbf{C}_{H}^{-1}+\overline{\mathbf{S}}_{D}^{H}[k] \overline{\mathbf{S}}_{D}[k]\right)^{-1}$. Using (14), $\mathbf{C}=\mathbf{D} \otimes \mathbf{I}_{N_{t}}$ via Kronecker product properties [18], and $\mathbf{D}=\left(N_{r} \sigma_{n}^{2} \mathbf{C}_{h}^{-1}+T E_{s} \mathbf{I}_{N}\right)^{-1}$ with the $(i, j)$-th entry $d_{i, j}$.
Therefore, dropping the time index for brevity, (16) can be written as

$$
\begin{aligned}
\hat{\overline{\mathbf{S}}} & =\underset{\overline{\mathbf{S}}}{\arg \max } \operatorname{tr}\left(\sum_{i=1}^{N} \sum_{j=1}^{N} d_{i, j} \mathbf{R}^{H}[i] \mathbf{S}[i] \mathbf{S}^{H}[j] \mathbf{R}[j]\right), \\
& =\underset{\overline{\mathbf{S}}}{\arg \max } \sum_{i=1}^{N} \sum_{j=1}^{N} d_{i, j} \operatorname{tr}\left(\mathbf{R}^{H}[i] \mathbf{S}[i] \mathbf{S}^{H}[j] \mathbf{R}[j]\right)
\end{aligned}
$$

where the second equality comes from the trace property. When $x_{p}$ belongs to a non-unitary constellation and $P$ is small so that (14) is not valid, an approximate ML detector for this case is given later in (24).

To further simplify (17), we note that $\operatorname{tr}(\mathbf{A B})=$ $\operatorname{vec}\left(\mathbf{A}^{H}\right)^{H} \operatorname{vec}(\mathbf{B})[18]$. For the $(i, j)$-th term

$$
\begin{aligned}
& d_{i, j} \operatorname{tr}\left(\mathbf{R}^{H}[i] \mathbf{S}[i] \mathbf{S}^{H}[j] \mathbf{R}[j]\right) \\
= & d_{i, j} \operatorname{vec}\left(\mathbf{S}^{H}[i] \mathbf{R}[i]\right)^{H} \operatorname{vec}\left(\mathbf{S}^{H}[j] \mathbf{R}[j]\right) .
\end{aligned}
$$

Substituting (3) into $\mathbf{S}^{H}[j] \mathbf{R}[j]$, we have

$$
\begin{aligned}
\operatorname{vec}\left(\mathbf{S}^{H}[j] \mathbf{R}[j]\right) & =\sum_{p=1}^{P} \alpha_{p}[j] \operatorname{vec}\left(\mathbf{A}_{p}^{T} \mathbf{R}[j]\right) \\
& -\jmath \beta_{p}[j] \operatorname{vec}\left(\mathbf{B}_{p}^{T} \mathbf{R}[j]\right)=\mathbf{F}_{j} \mathbf{s}[j],
\end{aligned}
$$

where $\mathbf{s}[j]=\left[\alpha_{1}[j], \ldots, \alpha_{P}[j], \beta_{1}[j], \ldots, \beta_{P}[j]\right]^{T}$ and $\quad \mathbf{F}_{j}=\left[\operatorname{vec}\left(\mathbf{A}_{1}^{T} \mathbf{R}[j]\right), \ldots, \operatorname{vec}\left(\mathbf{A}_{P}^{T} \mathbf{R}[j]\right)\right.$, $\left.-\jmath \operatorname{vec}\left(\mathbf{B}_{1}^{T} \mathbf{R}[j]\right), \ldots,-\jmath \operatorname{vec}\left(\mathbf{B}_{P}^{T} \mathbf{R}[j]\right)\right] . \quad$ Therefore, we can simplify (17) to derive the new blind detector

$$
\hat{\mathbf{s}}=\underset{\mathbf{s}}{\arg \max } \mathbf{s}^{T} \mathbf{G s},
$$

where $\mathbf{s}=\left[\mathbf{s}^{T}[1], \ldots, \mathbf{s}^{T}[N]\right]^{T}$ and $\mathbf{G}$ is a positive semidefinite block matrix with the $(i, j)$-th block $[\mathbf{G}]_{i, j}=d_{i, j} \mathbf{F}_{i}^{H} \mathbf{F}_{j}$.

If the channel coherence time is larger than $N T_{B}$, the channel remains constant during $N$ blocks. Using $M$-PSK constellations, all $d_{i, j}$ 's are then equal, and hence (17) reduces to the deterministic decision metric given in [9]-[11]. However, (17) is not limited to BPSK or QPSK as in [9]-[11].

Note that our blind detector (20) requires the knowledge of $\sigma_{n}^{2}$ and $\mathbf{C}_{h}$, which may not readily be obtained. They can be estimated using the detected data and estimated channel as shown in Section V. There may exist mismatch between the estimated $\sigma_{n}^{2}, \mathbf{C}_{h}$ and the true parameters. The investigation of parameters mismatch is beyond the scope of this paper.

\section{A. Efficient Detection}

For BPSK, (20) can be solved via semi-definite relaxation [15]. The idea is to relax (20) to a convex optimization problem called semidefinite programming (SDP) [15]. the blind ML detector output $\hat{\mathbf{s}}$ can be found via the GoemansWilliamson randomization [19], which provides good approximation accuracy with a modest number of randomization operations. The computational complexity of the whole semi-definite relaxation process including randomization is $O\left((N P)^{3.5}\right)$. However, the semi-definite relaxation detector is suboptimal. Consequently, we suggest the use of the sphere decoder [13] to attain ML performance. If $x_{p}[k]$ 's belong to unitary constellations, $\mathbf{s}^{T} \mathbf{S}=P N$ and $\eta \mathbf{s}^{T} \mathbf{S}$ is a constant, 
where $\eta$ is a constant. Therefore, the blind detector (20) can be restated as

$$
\hat{\mathbf{s}}=\underset{\mathbf{s}}{\arg \min } \mathbf{s}^{T}\left(\eta \mathbf{I}_{2 P N}-\mathbf{G}\right) \mathbf{s} .
$$

If $\eta$ is larger than the maximum eigenvalue of $\mathbf{G}, \rho_{\max }$, it can be readily verified that $\eta \mathbf{I}_{2 P N}-\mathbf{G}$ is positive definite. There are three possible choices of $\eta: \rho_{\max }+\sigma_{n}^{2}, \rho_{\max }+\rho_{\min }$, and $\operatorname{tr}(\mathbf{G})$, where $\rho_{\min }$ is the minimum non-zero eigenvalue of G. The third choice is valid since the matrix trace has the property [18]

$$
\operatorname{tr}(\mathbf{G})=\sum_{i=1}^{2 P N} \rho_{i}>\rho_{\max } .
$$

From our experimental experience, both the first and the second choices work well. However, the second choice needs additional complexity to compute the minimum non-zero eigenvalue of $\mathbf{G}$. Therefore, we use the first choice in the simulation.

To see how the blind detector (21) can be solved via the sphere decoder, we consider the Cholesky decomposition $\eta \mathbf{I}_{2 P N}-\mathbf{G}=\mathbf{M}^{T} \mathbf{M}$. The blind detector (21) can then be restated as

$$
\hat{\mathbf{s}}=\underset{\mathbf{s}}{\arg \min }\|\mathbf{M s}\|^{2} .
$$

Using the upper triangular structure of $\mathbf{M}$, the detector output (23) can be computed via the sphere decoder [13].

Note that for QPSK each element of $\mathbf{s}$ is independently chosen from the set $\{-1,1\}$. However, for $M$-PSK $(M>4)$, the real part $\alpha_{p}[n]$ and the imaginary part $\beta_{p}[n]$ cannot be chosen independently. In this case, if $\alpha_{p}[n]$ is chosen, $\beta_{p}[n]$ is restricted by the constellation. Such constellation-specific information can be incorporated into the sphere decoder. In the sphere decoder, when $\alpha_{p}[n]$ is assigned a value from its candidate set, the candidate set for $\beta_{p}[n]$ is determined by the bound given by the sphere decoder and the constellationspecific. The details of the complex sphere decoder are given in [20].

The blind detector (21) has also appeared in [10], [11] for blind OSTBC detection, and the sphere decoder is used to solve (21). However, the blind detector in [10], [11] is only applicable for BPSK and a static channel. References [10], [11] do not discuss how to choose $\eta$.

\section{B. Non-Unitary Constellations}

The determinant $\operatorname{det}\left(\mathbf{C}_{R}[k]\right)$ is not necessarily a constant when $x_{p}[k]$ 's are chosen from a non-unitary constellation such as quadrature amplitude modulation (QAM). Similarly, the superimposed pilots (Section IV-B) can be viewed as being chosen from a non-unitary constellation. This case may be handled by the use of the approximation (14) as done before. Alternatively, the term $\operatorname{det}\left(\mathbf{C}_{R}[k]\right)$ can be ignored (Section IV-B), and we solve (16) without the use of (14). Simulation results show that the performance loss due to ignoring $\operatorname{det}\left(\mathbf{C}_{R}[k]\right)$ is small. In order to develop the cost metrics for sphere decoding, we define $\xi_{\max }=\max \{|x| \mid x \in$ $\mathcal{Q}\}$ and $\xi_{\min }=\min \{|x| \mid x \in \mathcal{Q}\}$ where $\mathcal{Q}$ is the signal constellation. Eq. (16) is equivalent to minimizing

$$
g_{1}(\mathbf{s})=\eta P N-\operatorname{tr}\left(\overline{\mathbf{R}}^{H} \overline{\mathbf{S}}_{D}\left(N_{r} \sigma_{n}^{2} \mathbf{C}_{H}^{-1}+\overline{\mathbf{S}}_{D}^{H} \overline{\mathbf{S}}_{D}\right)^{-1} \overline{\mathbf{S}}_{D}^{H} \overline{\mathbf{R}}\right),
$$

where $\mathbf{s}$ is defined in (20). Let $\mathbf{A}=N_{r} \sigma_{n}^{2} \mathbf{C}_{H}^{-1}+\overline{\mathbf{S}}_{D}^{H} \overline{\mathbf{S}}_{D}$ and $\mathbf{B}=N_{r} \sigma_{n}^{2} \mathbf{C}_{H}^{-1}+T \xi_{\min }^{2} \mathbf{I}_{N}$. It can be readily verified that $\mathbf{A} \succeq \mathbf{B}$. Using Corollary [18, p. 471], it follows that $\mathbf{A}^{-1} \preceq \overline{\mathbf{B}}^{-1}$. We can prove that

$$
\operatorname{tr}\left(\overline{\mathbf{R}}^{H} \overline{\mathbf{S}}_{D} \mathbf{A}^{-1} \overline{\mathbf{S}}_{D}^{H} \overline{\mathbf{R}}\right) \leq \operatorname{tr}\left(\overline{\mathbf{R}}^{H} \overline{\mathbf{S}}_{D} \mathbf{B}^{-1} \overline{\mathbf{S}}_{D}^{H} \overline{\mathbf{R}}\right) .
$$

Therefore, the cost metrics are related as

$$
g_{1}(\mathbf{s}) \geq \mathbf{s}^{T}\left(\frac{\eta}{\xi_{\max }^{2}} \mathbf{I}_{2 P N}-\mathbf{G}^{\prime}\right) \mathbf{s}=g_{2}(\mathbf{s}),
$$

where the $(i, j)$-th block of $\mathbf{G}^{\prime}$ is $\left[\mathbf{G}^{\prime}\right]_{i, j}=b_{i, j} \mathbf{F}_{i}^{H} \mathbf{F}_{j}, b_{i, j}$ is the $(i, j)$-th entry of $\mathbf{B}$ and $\mathbf{F}_{i}$ is defined in (19). The sphere decoder uses the relation $g_{2}(\mathbf{s}) \leq g_{1}(\mathbf{s})<r^{2}$. All the candidates that satisfy $g_{2}(\mathbf{s})<r^{2}$ are found, and the one that makes $g_{1}(\mathbf{s})$ a minimum is the detector output. During the search, the bound (or the radius) $r^{2}$ can be updated by $g_{1}(\tilde{\mathbf{s}})$, where $\tilde{\mathbf{s}}$ is a valid candidate within the hyper-sphere. If $\xi_{\max }$ is much larger than $\xi_{\min }$, the bound given by $g_{1}(\mathbf{s})$ is loose and $g_{2}(\mathbf{s}) \leq r^{2}$ contains many points, which makes the algorithm inefficient.

\section{Totally Blind Detectors}

We consider the Alamouti code with $M$-PSK $\left(\mathcal{Q}_{M}=\right.$ $\left.\left\{e^{\jmath 2 \pi m / M}\right\}, m=0, \ldots, M-1\right)$ as an example. The results may be generalized to other OSTBC's and constellations (details omitted for brevity). We first provide a brief discussion on rotatable and non-rotatable OSTBCs and derive the dualconstellation and superimposed pilot schemes.

\section{A. Rotatable and Non-Rotatable OSTBCs}

A code is said to rotatable if for any codeword $\mathbf{S}$, there exists a unitary matrix $\Theta(\neq \pm \mathbf{I})$ such that $\mathbf{S} \Theta$ is also a valid codeword. Otherwise, the code is said to be non-rotatable [21]. Clearly, a rotatable code gives rise to a phase ambiguity in (17). For an optimal solution of (17) given by $\hat{\mathbf{S}}[i], i=$ $1, \ldots, N$, there may be a unitary matrix $\boldsymbol{\Theta}$ such that $\tilde{\mathbf{S}}[i]=$ $\hat{\mathbf{S}}[i] \boldsymbol{\Theta}$ is also an optimal solution of (17).

Non-rotatable OSTBCs are bandwidth efficient for blind decoding and need one pilot symbol only [21]. For example, the OSTBC in (41) is a non-rotatable code. In [21], a nonrotatable OSTBC criterion is proposed but it is only applicable to the BPSK constellation. We next give two conditions for a rotatable OSTBC.

Condition 1: An OSTBC is rotatable if for any permutation $\pi$ of $\{1, \ldots, P\}$, and $\pi \neq\{1, \ldots, P\}$, there exists a permutation matrix $\Pi$ satisfying

$$
\mathbf{C}_{i} \boldsymbol{\Pi}=a \mathbf{C}_{\pi(i)} \quad \text { and } \quad \mathbf{D}_{i} \boldsymbol{\Pi}=a \mathbf{D}_{\pi(i)}, \quad i=1, \ldots, P,
$$

where $\mathbf{C}_{i}$ and $\mathbf{D}_{i}$ are defined in (3) and $a= \pm 1$.

Condition 2: Let the set $I_{R}$ denote the index set of $\mathbf{C}_{i}$, $i=1, \ldots, P$, containing non-zero rows and the set $I_{C}$ denote the index set of $\mathbf{D}_{i}, i=1, \ldots, P$, containing non-zero rows. The code is rotatable only if

$$
I_{R} \cap I_{C}=\phi
$$

where $\phi$ is the null set.

The proof constitutes the fact that if these conditions are satisfied, then it is possible to construct a rotation matrix. 
For example, $I_{R}=\{1\}, I_{C}=\{2\}$, and $I_{R} \cap I_{C}=\phi$ for the Alamouti code. Therefore, it is rotatable and two possible rotation matrices are

$$
\boldsymbol{\Theta}=\left(\begin{array}{cc}
e^{\jmath 2 \pi k / M} & 0 \\
0 & e^{-\jmath 2 \pi k / M}
\end{array}\right), k \in\{0,1, \ldots, M-1\},
$$

and

$$
\boldsymbol{\Theta}=\left(\begin{array}{cc}
0 & -e^{\jmath 2 \pi k / M} \\
e^{-\jmath 2 \pi k / M} & 0
\end{array}\right), k \in\{0,1, \ldots, M-1\} .
$$

More generally, if both Conditions 1 and 2 are satisfied and all of the symbols are from an $M$-PSK constellation, the rotation matrix can be constructed as

$$
\Theta=\Lambda \Pi,
$$

where $\boldsymbol{\Lambda}$ is a diagonal matrix with diagonal entries

$$
[\boldsymbol{\Lambda}]_{i, i}=\left\{\begin{array}{cc}
e^{\jmath 2 \pi k / M} & i \in I_{R} \\
e^{-\jmath 2 \pi k / M} & i \in I_{C}
\end{array} .\right.
$$

If only Condition 1 is satisfied, we can set $\boldsymbol{\Lambda}=\mathbf{I}_{N_{t}}$. If only Condition 2 is satisfied, we can set $\boldsymbol{\Pi}=\mathbf{I}_{N_{t}}$. In either case, $\Theta$ defined in (31) is still a valid rotation matrix.

However, non-rotatable codes are rare as shown in [21] and they also need a pilot symbol. The use of pilot symbols results in a bandwidth loss, which motivates the research for totally blind detectors without any pilots. We present two such schemes next.

\section{B. Dual-constellation Scheme}

In [22], two different PSK-constellations are used to solve the phase ambiguity (equivalent to rotational and permutation ambiguities in this paper) in blind OFDM detection, which motivates our totally blind detector in this subsection. In the first scheme, we propose the use of dual constellations in $N$ consecutive blocks. The constellation design criterion is the following:

Criterion 1: Choose the two constellations $\mathcal{Q}^{1}$ and $\mathcal{Q}^{2}$ such that, for any $u \in \mathcal{Q}^{1}$ and for any $v \in \mathcal{Q}^{2}$, the phase angle of $u$ is different from the phase angle of $v$.

QPSK $\left(\mathcal{Q}_{4}=\left\{e^{\jmath m \pi / 2+\pi / 4}, m=0,1,2,3\right\}\right)$ and 3-PSK $\left(\mathcal{Q}_{3}=\left\{e^{j 2 m \pi / 3}, m=0,1,2\right\}\right)$ satisfy Criterion 1. For example, 3 -PSK is used in the $1,3, \ldots, N-1$ blocks and QPSK is used in the remaining blocks assuming $N$ is an even number. ${ }^{3}$ If $\hat{\mathbf{S}}[1]$ and $\hat{\mathbf{S}}[2]$ maximize (17), $\boldsymbol{\Theta}_{1}$ is a rotation matrix given by (29) or (30) for $\hat{\mathbf{S}}[1]$, and $\hat{\mathbf{S}}[1] \boldsymbol{\Theta}_{1}$ is also a feasible codeword. The rotation angle for $\hat{\mathbf{S}}[1]$ is a multiple of $\pi / 2$. However, when it is applied to $\hat{\mathbf{S}}[2]$, it can be verified that $\hat{\mathbf{S}}[2] \boldsymbol{\Theta}_{1}$ cannot result in valid codewords for $\hat{\mathbf{S}}[2]$ due to the use of different constellations. Similarly, the rotation matrix $\boldsymbol{\Theta}_{2}$ is also not applicable for $\hat{\mathbf{S}}[1]$. Thus, there does not exist a $\boldsymbol{\Theta}$ that makes both $\hat{\mathbf{S}}[1] \boldsymbol{\Theta}$ and $\hat{\mathbf{S}}[2] \boldsymbol{\Theta}$ valid. Therefore, (17) has a unique solution. QPSK with 5-PSK and 8-PSK with 7-PSK also satisfy the property.

\footnotetext{
${ }^{3}$ Note that the dual constellation scheme has also been applied to blind identification of the Alamouti code in [23]. However, in [23], the two symbols chosen from two different constellations are used within a single block, which are sent over the two transmitter antennas.
}

If the code has permutation ambiguity as opposed to Conditions 1 and 2, following the same argument as for the Alamouti code, it can be verified that it is a necessary condition to make the two different constellations satisfy Criterion 1 so that (17) results in a totally blind detector. Even though a OSTBC satisfies Conditions 1 and 2, our dual constellation scheme can also be applied. In this case, we do not need to insert any pilot, resulting in a bandwidth saving.

The 3-PSK and QPSK constellations pair is not optimized in [22]. We optimize the constellations by maximizing the Euclidean distance between the correct point and the wrong point induced by additive noise or phase ambiguity. We find that the optimal 3-PSK constellation is $\mathcal{Q}_{3}=\left\{1, e^{j 5 \pi / 8}, e^{-j 5 \pi / 8}\right\}$ and the optimal QPSK is $\mathcal{Q}_{4}=$ $\left\{e^{j k \pi / 2+\pi / 4}, k=0,1,2,3\right\}$.

The binary bits are mapped to 3-PSK via a punctured convolutional encoder in [22]. Here we introduce a mapping scheme similar to a linear block code. We map 3 binary bits to two 3-PSK symbols, which consists of 9 tuples. The tuple $(0,0)$ is not used and therefore we incur a loss of 0.17 bits. When performing ML detection, this tuple plays a role similar to that of the parity check bits in a linear block code, which enable error detection/correction. Since gray mapping does not exist for the 3-bit mapping, we develop a quasi-gray mapping scheme by minimizing the number of neighborhood bit errors. After optimization, we find that the suboptimal mapping scheme is given by (33) (see top of next page). The use of two PSK constellations reduces the minimum Euclidean distance. Alternatively, a semi-blind detector can be designed by transmitting one pilot (i.e., by fixing one element of s), which also eliminates the ambiguity problems. Compared with the detector with rotatable codes using a pilot block, the semiblind detector is also bandwidth efficient.

Using the dual-constellation scheme, the resulting totally blind detector (20) can be solved using the modified sphere detector for PSK in [20].

\section{Superimposed Pilot Scheme}

The superposition of pilot and data symbols has been proposed in [8], [24] for channel estimation. Our key idea is to use superimposed pilots to resolve the phase ambiguity. The $p$-th transmitted symbol in the $n$-th block can be represented as

$$
x_{p}[n]=\sqrt{\gamma_{n, p}} t_{p}[n]+\sqrt{\lambda_{n, p}} u_{p}[n],
$$

where $t_{p}[n]$ is the known pilot with power $E_{s}$ and $u_{p}[n]$ is a data symbol from $\mathcal{Q}$. We have $\gamma_{n, p}+\lambda_{n, p}$, and $\gamma_{n, p}$ denotes the percentage of the power allocated to training. In fact, (34) is a framework for all of the training schemes in this paper. If $\gamma_{n, p}=1$ for $p=1, \ldots, P$, it reduces to the case using a pilot block to solve the ambiguity. When $\gamma_{n, p}=0$ and the two constellations satisfying Conditions 1 and 2 are employed, it becomes the dual constellation scheme. We still call the detector using (34) a totally blind detector since if $\gamma_{n, p} \neq 1$, the data rate remains the same as $\gamma_{n, p}=0$ or full rate. The superimposed pilots can be used only for the first block, i.e., $0<\gamma_{1, p}<1$ and $\gamma_{n, p}=0$ for $n=2, \ldots, N$ or for all of the blocks $0<\gamma_{1, p}<1$ for $n=1, \ldots, N$. We show next that the 


$$
\begin{array}{lll}
100 \rightarrow\left(1, e^{j \frac{5 \pi}{8}}\right), & 010 \rightarrow\left(1, e^{-\jmath \frac{5 \pi}{8}}\right), & 001 \rightarrow\left(e^{\jmath \frac{5 \pi}{8}}, 1\right), \\
000 \rightarrow\left(e^{\frac{5 \pi}{8}}, e^{j \frac{5 \pi}{8}}\right), & 011 \rightarrow\left(e^{j \frac{5 \pi}{8}}, e^{-j \frac{5 \pi}{8}}\right), & 111 \rightarrow\left(e^{-\jmath \frac{5 \pi}{8}}, 1\right), \\
101 \rightarrow\left(e^{-j \frac{5 \pi}{8}}, e^{\frac{5 \pi}{8}}\right), & 110 \rightarrow\left(e^{-\jmath \frac{5 \pi}{8}}, e^{-\jmath \frac{5 \pi}{8}}\right) &
\end{array}
$$

superimposed pilots scheme is also a necessary condition for the totally blind detector.

The two constellations scheme resolves the ambiguity by modifying only the phase so that there does not exist a valid rotation matrix $\Theta$ for all of the OSTBC codewords from the two constellations. For constant $t_{p}[n]$, (34) forms a new nonsymmetric constellation for $x_{p}[n]$ with nonzero mean and we denote the new constellation as $\mathcal{Q}_{s}$. Clearly, either the phase or the amplitude of the point in $\mathcal{Q}_{s}$ are different from those in $\mathcal{Q}$. Except for BPSK, $\mathcal{Q}_{s}$ and $\mathcal{Q}$ satisfy Criterion 1. In addition, due to the difference in amplitude, the minimum Euclidean distance between the correct point and the wrong point by additive noise or phase ambiguity may be increased and this leads to performance improvement.

The value for $\gamma_{n, p}$ can be optimized. We take BPSK for example, and we assume that all the $\gamma_{n, p}$ 's are equal and $E_{s}=1$. If $\gamma_{n, p}>\lambda_{n, p}$ and $t_{p}[n]=1, \mathcal{Q}_{s}=\left\{\sqrt{\gamma_{n, p}}+\right.$ $\left.\sqrt{\lambda_{n, p}}, \sqrt{\gamma_{n, p}}-\sqrt{\lambda_{n, p}}\right\}$. Due to additive noise and permutation ambiguity, $\mathcal{Q}_{s}$ may be treated as $\mathcal{Q}_{s}^{\prime}=\left\{-\sqrt{\gamma_{n, p}}-\right.$ $\left.\sqrt{\lambda_{n, p}},-\sqrt{\gamma_{n, p}}+\sqrt{\lambda_{n, p}}\right\}$. To gain the best performance, we should maximize the minimum Euclidean distance in the set $\mathcal{Q}_{s} \cup \mathcal{Q}_{s}^{\prime}$, which results in

$$
\max _{\lambda_{n, p}, \gamma_{n, p}} \min \left\{2 \sqrt{\lambda_{n, p}}, 2 \sqrt{\gamma_{n, p}}-2 \sqrt{\lambda_{n, p}}\right\} .
$$

We can get $\gamma_{n, p}=\frac{4}{5}$ and $\lambda_{n, p}=\frac{1}{5}$. Similarly, if $\gamma_{n, p}<\lambda_{n, p}$, we have $\gamma_{n, p}=\frac{1}{5}$ and $\lambda_{n, p}=\frac{4}{5}$.

For the detection of symbols for the superimposed scheme, we note that if $\mathbf{S}[n]$ contains superimposed pilots, $\mathbf{S}^{H}[n] \mathbf{S}[n]$ cannot be approximated as $T E_{s} \mathbf{I}_{N_{t}}$ any more and $\mathcal{Q}_{s}$ is not a unitary constellation. We thus apply the modified sphere decoder (24)-(26) for non-unitary constellations. By choosing $\xi_{\max }$ and $\xi_{\min }$ for $\mathcal{Q}_{s}$, we can define $g_{2}(\mathbf{s})=\|\mathbf{M} \mathbf{s}\|^{2}$ as in (26), where $\mathbf{M}^{T} \mathbf{M}=\frac{\eta}{\xi_{\max }^{2}} \mathbf{I}_{2 P N}-\mathbf{G}^{\prime}$. Note that the $\mathbf{s}$ in (23) can be written as

$$
\mathbf{s}=\boldsymbol{\Gamma}_{t} \mathbf{t}+\boldsymbol{\Gamma}_{u} \mathbf{u},
$$

where $\mathbf{t}$ and $\mathbf{u}$ are formulated using the real and imaginary parts of $t_{p}[n]$ and $u_{p}[n]$ as $\mathbf{s}$ in (20), $\boldsymbol{\Gamma}_{t}$ and $\boldsymbol{\Gamma}_{u}$ are diagonal matrices with diagonal entries

$$
\begin{array}{r}
{\left[\boldsymbol{\Gamma}_{t}\right]_{(2 n-2) P+p,(2 n-2) P+p}=\left[\boldsymbol{\Gamma}_{t}\right]_{(2 n-1) P+p,(2 n-1) P+p}} \\
=\sqrt{\gamma_{n, p}}, n=1, \ldots, N, p=1, \ldots, P \\
{\left[\boldsymbol{\Gamma}_{u}\right]_{(2 n-2) P+p,(2 n-2) P+p}=\left[\boldsymbol{\Gamma}_{u}\right]_{(2 n-1) P+p,(2 n-1) P+p}} \\
=\sqrt{\lambda_{n, p}}, n=1, \ldots, N, p=1, \ldots, P
\end{array}
$$

Thus, $g_{2}(\mathbf{s})$ is reduced to

$$
g_{2}(\mathbf{u})=\left\|\mathbf{y}-\mathbf{M}^{\prime} \mathbf{u}\right\|^{2},
$$

where $\mathbf{y}=-\mathbf{M} \boldsymbol{\Gamma}_{t} \mathbf{t}$ and $\mathbf{M}^{\prime}=\mathbf{M} \boldsymbol{\Gamma}_{u}$. When using the sphere decoder, we solve $g_{2}(\mathbf{u}) \leq g_{1}(\mathbf{s})<r^{2}, g_{1}(\mathbf{s})$ is defined in (24) and $r^{2}$ is updated using $g_{1}(\mathbf{s})$.
Since the data symbols at the superimposed pilot blocks have less energy than other pure data symbols, they may not be reliably detected. To improve the overall performance, we first use the MMSE channel estimator in Section V to estimate the channel in pure data blocks. The channel at superimposed pilot blocks are then predicted and the superimposed data is detected using the linear coherent ML detector for an OSTBC.

\section{Channel Estimation and Prediction}

The "Parameters Estimator", "Channel Estimator and Predictor", and "Coherent Decoder" blocks in Fig. 1 are briefly discussed in this section. Although the data symbols can be efficiently detected using (20) without estimating the channel, the computational burden may be reduced by a dual-mode receiver with blind and decision-directed modes. This receiver starts with the blind mode using the blind detector (20) and then reverts to the decision-directed mode, where the detected symbols are used to predict the channel.

In the blind mode, after the data symbols have been detected using (20), the channel estimator may be designed according to the MMSE criterion using $\hat{\mathbf{S}}[k]$. With quasistatic fading (the case of static fading can be obtained similarly.), the MMSE channel estimator is given by

$$
\hat{\mathbf{H}}=\left(\hat{\mathbf{S}}_{D}^{H} \hat{\mathbf{S}}_{D}+\sigma_{n}^{2}\left(\mathbf{C}_{h}^{-1} \otimes \mathbf{I}_{N_{t}}\right)\right)^{-1} \hat{\mathbf{S}}_{D}^{H} \overline{\mathbf{R}},
$$

where $\overline{\mathbf{H}}=\left[\mathbf{H}^{T}[1], \mathbf{H}^{T}[2], \ldots, \mathbf{H}^{T}[N]\right]^{T}$ as defined in (10). To bound the performance of the proposed blind receiver and to optimize the power allocation between pilot symbols and data symbols, we derive the CRB of the channel estimate for the joint channel estimation and detection following [25], which assumes the entries of $\mathbf{S}[k]$ are Gaussian distributed. ${ }^{4}$ After a lengthy derivation of the Fisher Information Matrix (FIM) [25], we derive the CRB as

$$
\begin{aligned}
\mathrm{CRB} & =N_{r} \operatorname{tr}\left(\left(\frac{1}{\sigma_{n}^{2}} \mathbf{E} \otimes \mathbf{I}_{N_{t}}+\mathbf{C}_{h}^{-1} \otimes \mathbf{I}_{N_{t}}\right)^{-1}\right), \\
& =N_{r} N_{t} \operatorname{tr}\left(\left(\frac{1}{\sigma_{n}^{2}} \mathbf{E}+\mathbf{C}_{h}^{-1}\right)^{-1}\right)
\end{aligned}
$$

where $\mathbf{E}=\operatorname{diag}\left\{\mathcal{E}_{1}, \ldots, \mathcal{E}_{N}\right\}$, and $\mathcal{E}_{i}$ is the total power assigned to the $i$-th block. When superimposed pilots are used, a remarkable property of (40) is that the CRB does not depend on the power allocated to the pilots or the location of pilots due to the property of OSTBC. However, the training power determines the SNR required to achieve the CRB. The more training power, the lower SNR is needed. All the power allocation schemes achieve the CRB in high SNR.

The CRB for quasistatic fading channels (40) only depends on the total power allocated to each block. When the channel

\footnotetext{
${ }^{4}$ Note that even though the entries of $\mathbf{S}[k]$ are of discrete values, this Gaussian simplification provides a tractable lower bound on the performance.
} 
is almost constant during the $N$ blocks, from an information point of view, the optimal power allocation scheme is $\mathcal{E}_{k+1}=\mathcal{E}_{k+2}=\ldots \mathcal{E}_{k+N}=\mathcal{E} / N$ by maximizing the mutual information between the input and the output [24]. But, when the channel is time-selective, the optimal power allocation for each block should minimize the CRB (40) given constant total power $\mathcal{E}$. In practice, to simplify system design, one may nevertheless employ the equal power allocation scheme.

In the decision-directed mode, Wiener filtering or Kalman filtering [26] may be applied to predict the channel in the following blocks if the channel correlation is known at the receiver. With $\hat{\mathbf{H}}[N+1]$, the data symbol in the $N+1$ th block can then be decoded using the linear ML detector. The decoded symbol $\hat{\mathbf{S}}[N+1]$ can be used to update the channel estimate $\hat{\mathbf{H}}[N+1]$ using the MMSE channel estimator (39) with $N=1$. In the $N+2$-th block, the channel is first predicted using the estimated channels from the 2nd to the $N+1$-th blocks. Similarly, the data symbols are first decoded using the predicted channel and are then used to update the channel estimate. The decision-directed mode lasts for $N_{d}$ blocks. The receiver then reverts to blind mode to stop the error propagation caused by the decision-direct. This will alleviate the computational complexity of the blind detector since the linear ML detector has low complexity. The bandwidth efficiency is also improved since the receiver can work in full rate transmission without transmitting pilots.

Note that in both modes, the blind detector (20) and the MMSE channel estimator need the knowledge of $\sigma_{n}^{2}$ and $\mathbf{C}_{h}$. If $\overline{\mathbf{S}}[k]$ is known, (8) can also be considered as an ML function of $\sigma_{n}^{2}$ and $\mathbf{C}_{h}$. But (8) is a nonlinear function in $\sigma_{n}^{2}$ and $\mathbf{C}_{h}$. It is complicated to estimate them directly from (8). At the receiver, $\sigma_{n}^{2}$ and $\mathbf{C}_{h}$ can also be estimated using the time average of $\|\mathbf{R}[n]-\hat{\mathbf{S}}[n] \hat{\mathbf{H}}[n]\|^{2}$ and $\hat{\mathbf{H}}^{H}[n] \hat{\mathbf{H}}[n]$. Therefore, the blind detector and channel estimator, as a whole, form a bandwidth efficient and low complexity receiver structure.

\section{Simulation Results}

We now present simulation results for our blind detector over a flat Rayleigh fading channel. For more realistic results, rather than simply using the quasistatic fading model, we generate the MIMO channel gains by sampling a continuous fading process via the Jakes model [17]. The SNR is defined to be $E\left\{\|\mathbf{H}\|_{F}^{2}\right\} / \sigma_{n}^{2}$. As well, it is assumed that the receiver has perfect knowledge of channel correlation and noise variance. We first compare our proposed blind detector with those proposed in the literature [7]-[9] in terms of both performance and complexity. Note those detectors need pilots to resolve the ambiguity. The cyclic ML is initialized by a pilot symbol according to the Blind Detector in [7]. We next show the performance of our blind receiver working in both blind and decision-directed modes. Finally, we compare our proposed totally blind detectors.

\section{A. Comparison of Different Blind OSTBC Detectors}

We first consider the non-rotatable OSTBC with $N_{t}=3$ and $P=4$ [9]

$$
\left(\begin{array}{cccc}
x_{1} & x_{2} & x_{3} & x_{4} \\
-x_{2} & x_{1} & x_{4} & -x_{3} \\
-x_{3} & -x_{4} & x_{1} & x_{2}
\end{array}\right) .
$$

The number of receiver antennas is $N_{r}=3$ and the number of blocks is $N=8$. BPSK is used for this code. $x_{1}[1]$ is transmitted as a pilot to solve the phase ambiguity. The MATLAB V5.3 command "flops" is used to count the number of flops. The ML detection with perfect CSI is used as the benchmark. The semi-definite relaxation algorithm follows exactly the one given in [15].

We first consider that the channel remains constant for $N$ blocks. Fig. 2 shows the BER versus SNR of various blind detectors, i.e., (23) with sphere decoder (stochastic SD), deterministic SDR [9], blind cyclic [7] and blind subspace [8]. The stochastic SD and deterministic SDR perform substantially better than the other blind detectors. At a BER of $10^{-3}$, the stochastic SD has a $0.2-\mathrm{dB}$ gain over the deterministic SDR. Although the stochastic SD performs $2 \mathrm{~dB}$ worse than the benchmark, it has a smaller performance loss and complexity than differential unitary space time modulation (DUSTM) [27], where a 3-dB loss is observed. Our stochastic SD achieves the same diversity order as the benchmark.

Figs. 3 and 4 compare the average complexities of different blind detectors in a static channel. We simulate this system using MATLAB V7.0 on a PC with an Intel Pentium-4 processor at $3.4 \mathrm{GHz}$. Both the average flop count and the average computational time are used as the complexity measures. The complexity of the preprocessing stage such as Cholesky decomposition in the stochastic SD or the initialization in [7], [8] is also counted. The complexities of deterministic SDR, blind subspace, and blind cyclic are almost independent of SNR, while that of blind SD depends on SNR. In terms of flops, the deterministic SDR is the most complex one, while the proposed stochastic SD is the least complex. Therefore, the blind SD outperforms the other blind detectors in terms of both BER and complexity. However, when the average computational time is measured, the blind subspace achieves the minimum complexity. The stochastic SD only has less computational time in high SNR. A possible explanation is the fact that the deterministic SDR, blind subspace, and blind cyclic detectors can exploit Matlab vector operation, whereas the stochastic SD does not. Thus, on processors that have special hardware (parallel) architectures for vector and matrix operations, matrix-based algorithms may be faster than the sphere decoder.

The effects of channel variation on different detectors are shown in Fig. 5 for $f_{d} T_{B}=0.04$. The stochastic SDR denotes solving (20) using semi-definite relaxation. The deterministic SDR, blind cyclic and blind subspace detectors perform poorly due to the model mismatch. At a BER of $10^{-2}$, the stochastic SD still has a 0.2-dB gain over the stochastic SDR. However, the performance gap between stochastic SD and the benchmark increases to $2.8 \mathrm{~dB}$, since the quasistatic assumption is invalid for a large Doppler spread. Our blind detector (20) also shows a model mismatch problem.

\section{B. Performance of Totally Blind Detectors}

We next test our totally blind detector for rotatable Alamouti code (2). The number of receiver antennas is $N_{r}=2$ and the number of blocks is $N=4$. We compare the performance of a training based detector (TRD) with a pilot block, a totally 


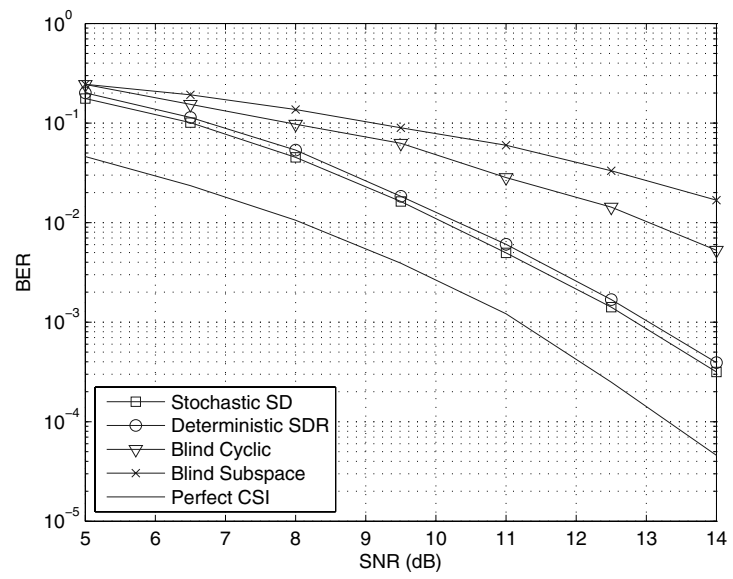

Fig. 2. BER versus SNR for different blind detectors with $N=8$ and BPSK over a static channel.

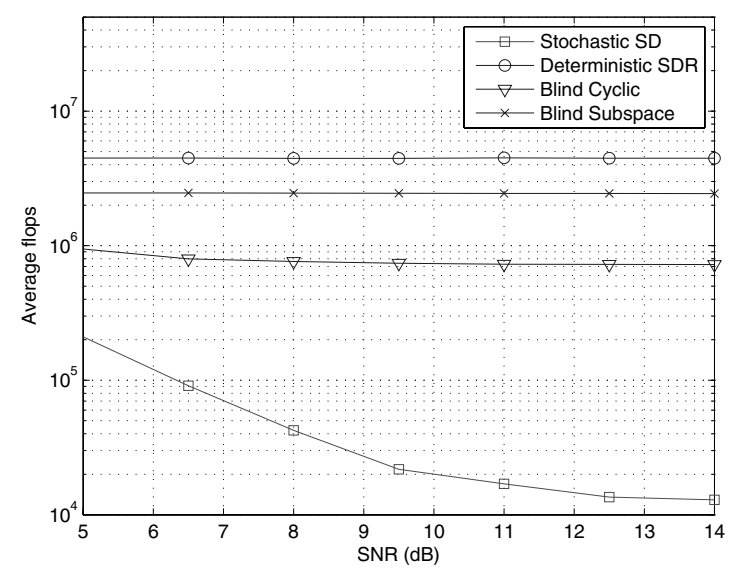

Fig. 3. Average flops versus SNR for different blind detectors with $N=8$ and BPSK over a static channel.

blind detector with dual constellations (TCD) and a totally blind detector with superimposed pilots (SPD). In the TRD, QPSK is used and the first block contains all pilots. QPSK and 3-PSK are used for TCD. The first and third blocks use 3-PSK and the second and fourth blocks use QPSK. The 3-PSK signal points are mapped using (33). In SPD, superimposed BPSK pilots and data are used for the first block, and $\gamma_{1, p}=\frac{4}{5}$, $\lambda_{1, p}=\frac{1}{5}$ and $t_{p}[1]=1, p=1,2$. The remaining blocks use QPSK. We also show the performance for semi-blind TCD with one pilot and denote it as TCD-SB. TRD transmits 12 bits, TCD 14 bits, SPD 14 bits and TCD-SB 13 bits in the $N$ blocks. To compare the performance of detectors with different data rates, we use the effective signal-to-noise ratio per data bit:

$$
\mathrm{SNR}_{b}=\frac{N E\left\{\|\mathbf{H}\|_{F}^{2}\right\}}{N_{b} \sigma_{n}^{2}},
$$

where $N_{b}$ is the number of bit transmitted in $N$ blocks.

Figs. 6 and 7 compare the BER and MSE performance of different detectors in a time-selective channel with $f_{d} T_{B}=$ 0.005. The TCD performs the worst and has a 4-dB loss over the TRD at a BER of $10^{-3}$. However the TCD-SB gains 0.8$\mathrm{dB}$ over the TCD. The SPD performs better than both the TCD

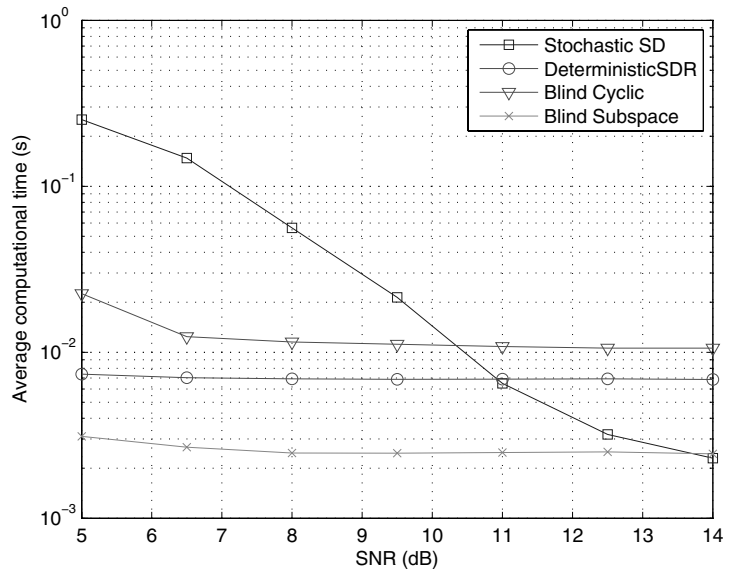

Fig. 4. Average computational time versus SNR for different blind detectors with $N=8$ and BPSK over a static channel.

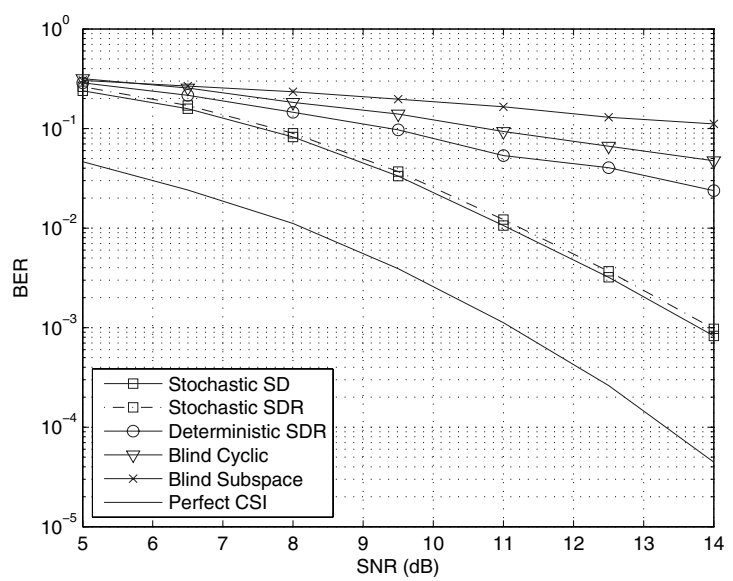

Fig. 5. BER versus SNR for different blind detectors with $N=8$ and BPSK over a time-selective channel with $f_{d} T_{B}=0.04$.

and TCD SB. Even though SPD has a 2.5-dB loss over TRD, two more bits are transmitted using SPD, which gives a $17 \%$ bandwidth improvement. The MSE (43) of channel estimation is shown in Fig. 7. The different detectors are compared in terms of the SNR per bit, and their CRB's are different. SPD-DD denotes the performance of channel estimation using the decision-directed mode. Although the channel is assumed quasistatic for the blind detectors and channel estimators, they can achieve their corresponding CRBs in high SNR. SPD and TCD-SB require a lower SNR to achieve the CRB than TCD. This also justifies that when $f_{D} T_{B}$ is small, the time-selective channel becomes quasistatic. When the Doppler spread is large, the quasistatic assumption becomes invalid, and our blind detectors perform worse. Nevertheless, they perform well provided the Doppler spread is small.

\section{Performance of Dual-Mode Blind Receivers}

Figs. 8 and 9 present the BER and MSE of of a receiver working in blind mode (BM) and decision-directed mode (DDM). The number of blocks in decision-directed mode is chosen as $N_{d}=4,8,16$. The system parameters are the same 


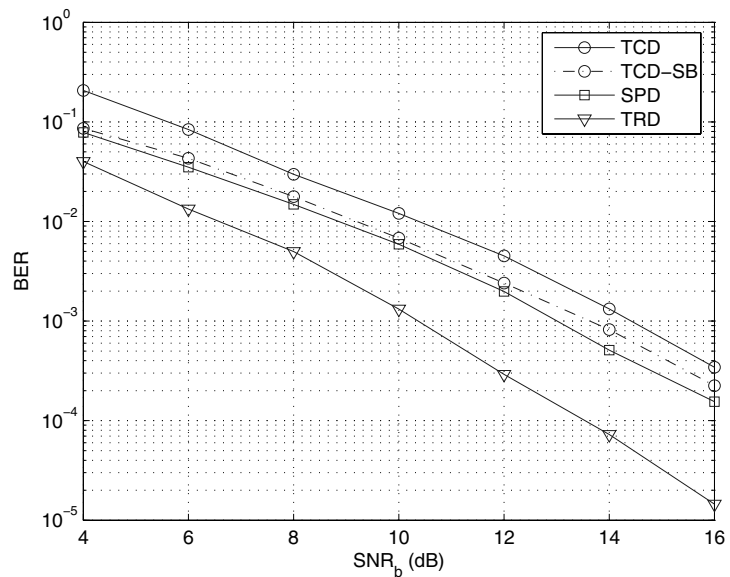

Fig. 6. BER performance of different totally blind detectors in a timeselective channel with $f_{d} T_{B}=0.005$.

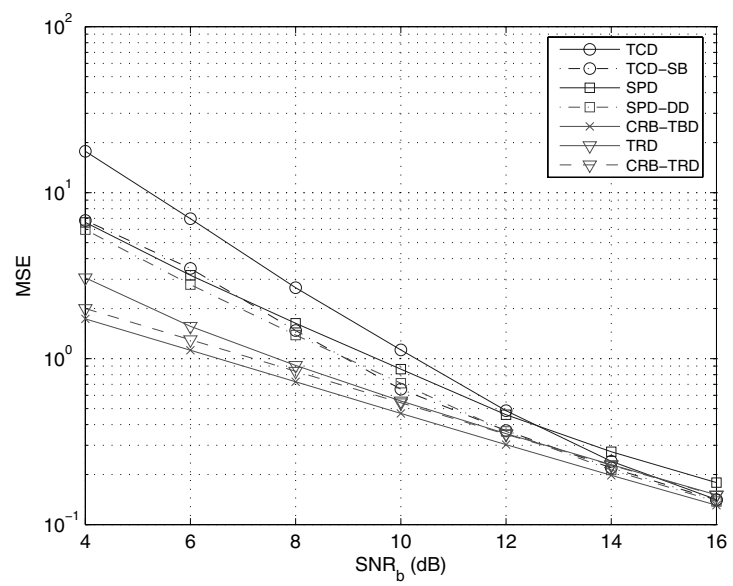

Fig. 7. MSE performance of different totally blind detectors in a timeselective channel with $f_{d} T_{B}=0.005$.

as before and $f_{d} T_{B}=0.02$. The MSE of channel estimate is defined as

$$
\mathrm{MSE}=\frac{E\left\{\sum_{t=1}^{T}\|\mathbf{H}[t]-\hat{\mathbf{H}}\|_{F}^{2}\right\}}{T},
$$

where $\mathbf{H}[t]$ is the MIMO channel during the $t$-th symbol in each block. When $N_{d}$ increases, the BER of DDM increases due to the error propagation caused by the decision-direct mode. However, all the DDMs perform within $0.6 \mathrm{~dB}$ of the BM. But when $N_{d}=16$, the dual-mode receiver has a $66 \%$ reduction in complexity over the BM receiver. In practical systems, the performance loss due to the increase of $N_{d}$ may be compensated by error correction coding. Fig. 9 shows the MSE of channel estimation. DDM-PR denotes the channel predictor using MMSE and DDM-DD is the channel estimator using the decision-direct mode. With different $N_{d}$, all the cases achieve almost the same MSE with DDM-PR.

\section{CONCLUSIONS}

We considered stochastic blind maximum-likelihood detection of OSTBCSs over a quasi-static flat MIMO Rayleigh

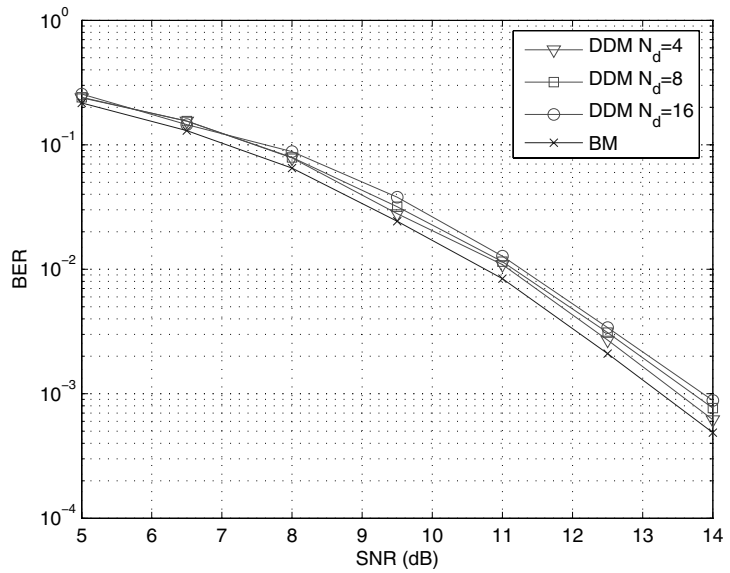

Fig. 8. BER performance of a blind receiver working two modes with different $N_{d}$ and $f_{d} T_{B}=0.02$.

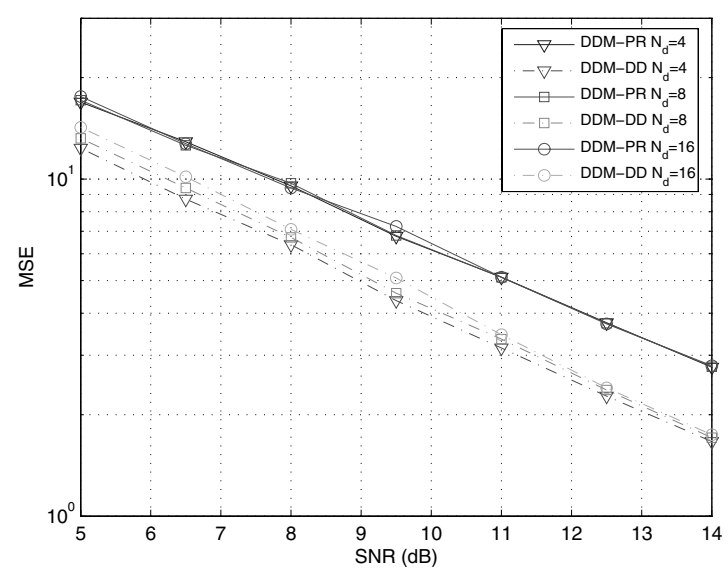

Fig. 9. MSE performance of a blind receiver working two modes with different $N_{d}$ and $f_{d} T_{B}=0.02$.

fading channel. A general decision rule for stochastic blind maximum-likelihood OSTBC detection has been derived. The blind detectors have been implemented by semi-definite relaxation or sphere decoding. For the latter, the modifications necessary for both unitary and non-unitary constellations have been developed. Two totally blind detectors using dual constellations or a superimposed training scheme were proposed. As a side product, two conditions for a rotatable OSTBC were also derived. A decision-directed, minimum mean-squareerror (MMSE) channel estimator was also developed. The Cramér-Rao bound (CRB) for channel estimation was derived. Simulation results show that our proposed blind detector with SD achieves better performance than other blind detectors but with less complexity. The blind dual-mode receiver not only performs well, but also reduces complexity.

\section{ACKNOWLEDGMENT}

The authors would like to thank the anonymous reviewers and the associate editor for their critical comments that greatly improved this paper. 


\section{REFERENCES}

[1] S. M. Alamouti, "A simple transmit diversity technique for wireless communications," IEEE J. Select. Areas Commun., vol. 16, no. 8, pp. 1451-1458, Oct. 1998.

[2] V. Tarokh, H. Jafarkhani, and A. Calderbank, "Space-time block coding for wireless communications: Performance results," IEEE J. Select. Areas Commun., vol. 17, no. 3, pp. 451-460, Mar. 1999.

[3] — - "Space-time block codes from orthogonal designs," IEEE Trans. Inform. Theory, vol. 45, no. 5, pp. 1456-1467, July 1999.

[4] F.-C. Zheng and A. Burr, "Orthogonal space-time block coding over time-selective fading channels: A PIC detector for the H/sub i/ systems," in Proc. IEEE ICC, June 2004, pp. 687-691.

[5] - "Signal detection for non-orthogonal space-time block coding over time-selective fading channels," IEEE Commun. Lett., vol. 8, no. 8, pp. 491-493, Aug. 2004.

[6] - "Signal detection for orthogonal space-time block coding over time-selective fading channels: A PIC approach for the G/sub i/ systems," IEEE Trans. Commun., vol. 53, no. 6, pp. 969-972, June 2005.

[7] P. Stoica and G. Ganesan, "Space-time block codes: Trained, blind and semi-blind detection," in Proc. IEEE ICASSP, May 2002, pp. 7-11.

[8] A. L. Swindlehurst and G. Leus, "Blind and semi-blind equalization for generalized space-time block codes," IEEE Trans. Signal Processing, vol. 50, no. 10, pp. 2489-2498, Oct. 2002.

[9] W.-K. Ma, P. C. Ching, T. N. Davidson, and X. G. Xia, "Blind maximum-likelihood decoding for orthogonal space-time block codes: A semidefinite relaxation approach," in Proc. IEEE GLOBECOM, Dec. 2003, pp. 2094-2098.

[10] W.-K. Ma, B.-N. Vo, T. Davidson, and P. Ching, "On implementing the blind ML receiver for orthogonal space-time block codes," in Proc. IEEE ICASSP, Mar. 2005, pp. iii/437-iii/440.

[11] W.-K. Ma, B.-N. Vo, T. Davidson, and P.-C. Ching, "Blind ML detection of orthogonal space-time block codes: Efficient high-performance implementations," IEEE Trans. Signal Processing, vol. 54, no. 2, pp. 738-751, Feb. 2006

[12] R. Schober and L. H. J. Lampe, "Noncoherent receivers for differential space-time modulation," IEEE Trans. Commun., vol. 50, no. 5, pp. 768777, May 2002.

[13] U. Fincke and M. Pohst, "Improved methods for calculating vectors of short length in a lattice, including a complexity analysis," Math. Computation, vol. 44, pp. 463-471, Apr. 1985.

[14] C. P. Schnorr and M. Euchner, "Lattice basis reduction: Improved practical algorithms and solving subset sum problems," Math. Programming, vol. 66, pp. 181-191, 1994.

[15] C. Helmberg, F. Rendl, R. J. Vanderbei, and H. Wolkowicz, "An interior point method for semidefinite programming," SIAM J. Optim., vol. 6, no. 2, pp. 342-361, May 1996.

[16] B. Hassibi and B. M. Hochwald, "High-rate codes that are linear in space and time," IEEE Trans. Inform. Theory, vol. 48, no. 7, pp. 1804-1824, July 2002.

[17] J. W. C. Jakes, Microwave Mobile Communications. New York: Wiley, 1974.

[18] R. A. Horn and C. R. Johnson, Matrix Analysis. Cambridge/New York: Cambridge University Press, 1985.

[19] M. X. Goemans and D. P. Williamson, "Improved approximation algorithms for maximum cut and satisfiability problem using semi-definite programming," J. ACM, vol. 42, pp. 1115-1145, Nov. 1995.

[20] T. Cui and C. Tellambura, "Joint channel estimation and data detection for OFDM systems via sphere decoding," in Proc. IEEE GLOBECOM, Dec. 2004, vol. 6, pp. 3656-3660.
[21] W.-K. Ma, P. Ching, T. Davidson, and B.-N. Vo, "Blind symbol identifiability of orthogonal space-time block codes," in Proc. IEEE ICASSP, May 2004, pp. iv/821-iv/824.

[22] M. C. Necker and G. L. Stüber, "Totally blind channel estimation for OFDM on fast varying mobile radio channels," IEEE Trans. Wireless Commun., vol. 3, no. 5, pp. 1514-1525, Sep. 2004.

[23] L. Zhou, J.-K. Zhang, and K. Wong, "Blind unique identification of Alamouti space-time coded channel via signal design and transmission technique," in Proc. International Symposium Signal Processing Applications, Aug. 2005, pp. 691-694.

[24] C. Budianu and L. Tong, "Channel estimation for space-time orthogonal block codes," IEEE Trans. Signal Processing, vol. 50, no. 10, pp. 25152528, Oct. 2002.

[25] M. Dong and L. Tong, "Optimal design and placement of pilot symbols for channel estimation," IEEE Trans. Signal Processing, vol. 50, no. 12, pp. 3055-3069, Dec. 2002.

[26] S. Gazor and H. Rad, "Space-time coding ambiguities in joint adaptive channel estimation and detection," IEEE Trans. Signal Processing, vol. 52, no. 2, pp. 372-384, Feb. 2004.

[27] B. M. Hochwald and W. Sweldens, "Differential unitary space-time modulation," IEEE Trans. Commun., vol. 48, no. 12, pp. 2041-2052, Dec. 2000.

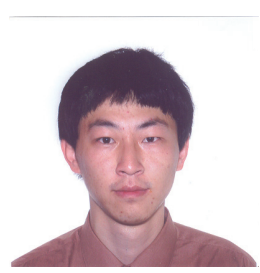

Tao Cui (S'04) received the B.Eng. degree in information engineering from Xi' an Jiaotong University, Xi' an, China, in 2003, and the M.Sc. degree from the University of Alberta, Edmonton, AB, Canada in 2005. He is currently working toward the Ph.D. degree in the Department of Electrical Engineering, California Institute of Technology, Pasadena, CA, USA.

His research interests are in communication theory, broadband wireless communications, space-time coding, MIMO systems and wireless networks. He is a past recipient of postgraduate scholarships from the Alberta Ingenuity Fund and the Alberta Informatics Circle of Research Excellence (iCORE).

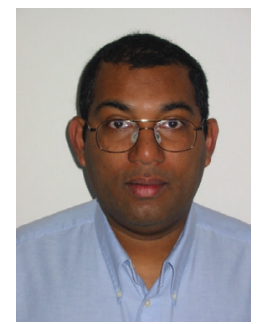

Chintha Tellambura (SM'02) received the B.Sc. degree (with first-class honors) from the University of Moratuwa, Moratuwa, Sri Lanka, in 1986, the M.Sc. degree in electronics from the University of London, London, U.K., in 1988, and the $\mathrm{Ph} . \mathrm{D}$. degree in electrical engineering from the University of Victoria, Victoria, BC, Canada, in 1993.

He was a Postdoctoral Research Fellow with the University of Victoria (1993-1994) and the University of Bradford (1995-1996). He was with Monash University, Melbourne, Australia, from 1997 to 2002. Presently, he is a Professor with the Department of Electrical and Computer Engineering, University of Alberta. His research interests include coding, communication theory, modulation, equalization, and wireless communications.

Prof. Tellambura is an Associate Editor for both the IEEE TRANSACTIONS ON COMMUNICATIONS and the IEEE TRANSACTIONS ON WIRELESS COMMUNICATIONS. He was Chair of the Communication Theory Symposium in Globecom'05 held in St. Louis, MO. 